\title{
Gorongosa and Sasagamine: intra-species behavioral variation in baboons and Japanese monkeys
}

\author{
Tetsuro Matsuzawa ${ }^{1,2,3}$
}

Published online: 7 November 2018

(c) Japan Monkey Centre and Springer Japan KK, part of Springer Nature 2018

In August 2018, I had the opportunity to spend a week in Gorongosa National Park in Mozambique. Mozambique, formerly a Portuguese colony, gained independence in 1975. During the civil war that followed (1977-1992), animals in Gorongosa were targeted by soldiers as food. After the war, the number of animals started to recover, partly through a reintroduction program sponsored by the Carr Foundation, founded by Mr. Gregory Carr (Wilson 2014).

Gorongosa National Park currently covers $3770 \mathrm{~km}^{2}$. Tokyo prefecture is $2188 \mathrm{~km}^{2}$, and is home to about 10 million people. Therefore, Gorongosa is close to 1.8 times the size of Tokyo prefecture, but has no large human settlements. We traveled around the park by helicopter to get a sense of its scale. With the doors of the helicopter removed, we got an excellent view of the landscape (Fig. 1). We saw a mosaic of savanna and woodland, and we could see the horizon far in the distance: the land was flat, the rivers winding. Because of the noise of the helicopter, many of the animals broke into a run. We saw large herds of waterbuck, the most populous species in the park. The estimated number of waterbuck in 2016 was about 45,000 individuals, according to the Park's records. From the sky we also saw elephants, hippos, crocodiles, various species of antelopes, and many more animals.

We also went on a safari drive early one morning when it was very cool. We encountered many impalas, warthogs, baboons, and other species (Fig. 2). There was a flock of vultures eating the remaining fresh meat off an impala's carcass that had probably been killed by lions the previous night. On the return journey, the air heated up. We encountered two groups of elephants staying near to trees for shade. That

Tetsuro Matsuzawa

matsuzawa.tetsuro.8w@kyoto-u.ac.jp

Kyoto University Institute for Advanced Study, Kyoto, Japan

2 Primate Research Institute, Kyoto University, Inuyama, Japan

3 Japan Monkey Centre, Inuyama, Japan

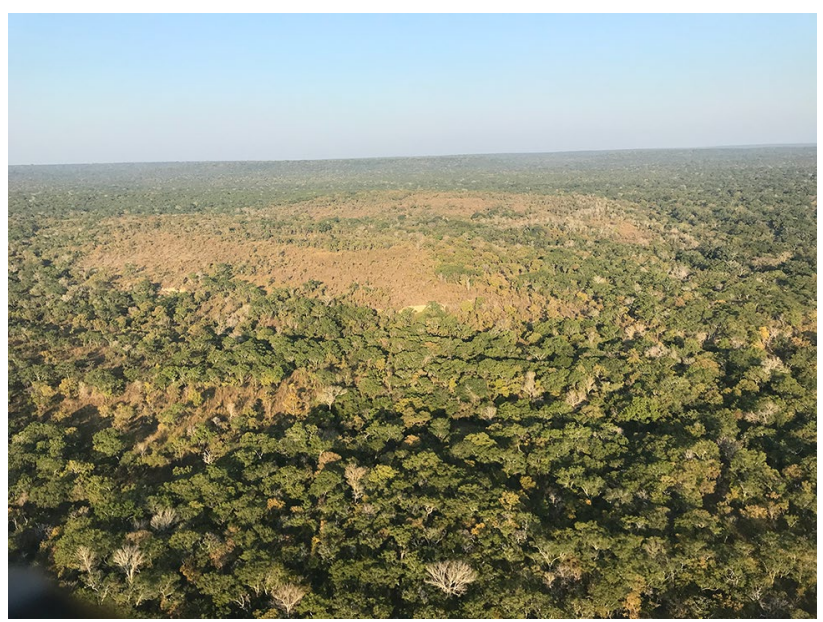

Fig. 1 The view of Gorongosa National Park from the sky (photo taken by Tetsuro Matsuzawa)

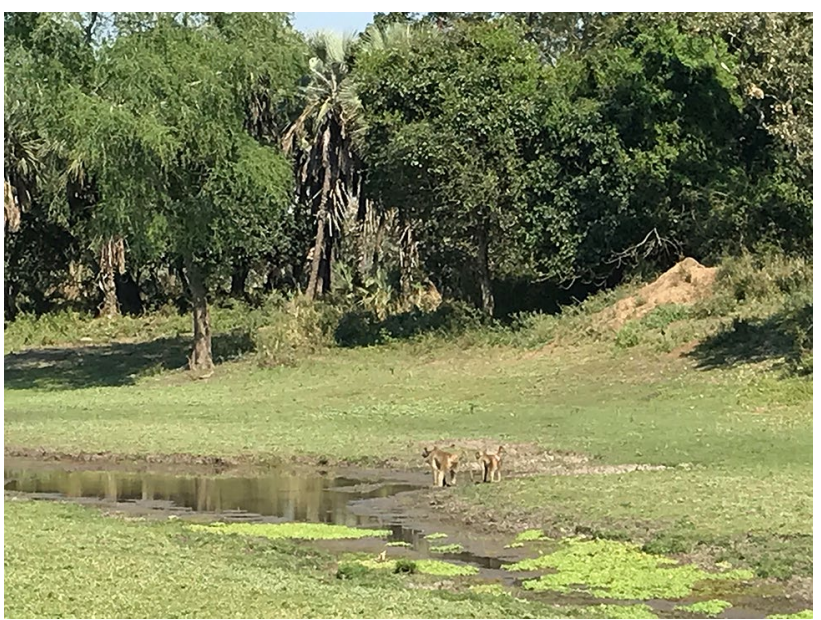

Fig. 2 A consort pair of chacma baboons in Gorongosa National Park (photo taken by Tetsuro Mastuzawa) 
scene reminded me of the feral horses I met in Serra d'Arga in northern Portugal (Matsuzawa 2017). The two species rest in the shade similarly, both making tandem lines.

The field study of primates at Gorongosa is run by two Oxford scholars, Susana Carvalho and Dora Biro, both exstudents of mine. They invited me to accompany them on their field research. Guided by their PhD students, I had the opportunity to observe two troops of chacma baboons (Papio ursinus). The two troops are separated from each other only by a few kilometers, but live in very different environments: One lives in the woodland, the other on a grassland flood plain that it shares with waterbuck, impala, and even lions.

The flood plain where the grassland troop lives will turn into a huge lake in the rainy season. What will happen then? We do not know the details yet, but the baboons must migrate from the flood plain to the neighboring dry area. Their annual activity cycle is waiting to be studied.

Baboons, the genus Papio, are predominant across Africa and the southern part of the Arabian Peninsula, inhabiting various environments across the continent. There is another successful group of nonhuman primates that adapts to various environments, namely macaques, the genus Macaca.

I got a job in the Primate Research Institute in 1976. During the first winter, I went to Shiga Heights to see wild Japanese monkeys, often referred to as snow monkeys (Macaca fuscata). I studied the wild monkeys there (Matsuzawa et al. 1983; Wada and Mastzuawa 1986). In 1977 I started to focus on chimpanzee research in the laboratory and in the wild. However, I tried to take opportunities to visit the natural habitats of Japanese snow monkeys. Starting in the north, I have been to Shimokita Peninsula, Shiragami Mountains, Kinkazan Island, Shiga Heights, Myoko Heights, Kurobe valleys, Mt. Hakusan, Arashiyama in Kyoto, Shodoshima Island, Kohshima Island, and Yakushima Island. Thus, I have visited wild monkeys that have adapted to living in 11 different environments. The variation in behavior within the same species is enormous. Depending on where they live, the monkeys love to wash sweet potatoes (Schofield et al. 2018), bathe in a hot spring in Shiga Heights (Matsuzawa 2018), form extra-large clusters in Shodoshima (Zhang et al. 2007), and ride on deer in Yakushima (Pelé et al. 2017).

The two troops of baboons in Gorongosa reminded me of the Japanese monkeys in Myoko Heights. I belong to the Kyoto University Alpine Club (KUAC), which has a hütte in Sasagamine, located in the heart of Myoko Heights. Almost every year since 1969 , when I first entered university, I have visited Sasagamine hütte. During this half-century, the environment of Sasagamine has gradually changed. One feature is the decreasing population in the nearby villages. We saw many more wild monkeys on the roads year by year. Another feature is the changing landscape. Sasagamine used to be a green meadow where we saw cows and sheep. However, the meadows have been shrinking and the forests have regained a hold. The forests are mixed with various kinds of deciduous trees such as oak, Japanese beech, Betulas, etc. In the past 5 years, we started to see the monkeys at Sasagamine hütte, at an altitude of $1300 \mathrm{~m}$. During my recent visits in September and October 2018, I saw monkeys every day (Fig. 3). They have successfully expanded into the new habitat of the Sasagamine area.

In the genus Macaca, the rhesus monkey (Macaca mulatta) has the largest geographic distribution, occurring from Afghanistan to China via India. The eastern limit of the distribution of Macaca is the Japanese monkey. The western limit is a species sometimes called the Barbary ape (Macaca sylvanus); due to its very short tail, it lives in the northern part of Africa, not far from the Mediterranean. Thus, a large area of the Old World, meaning Asia and Africa in this case, seems to be occupied by baboons from the west and macaques from the east.

The common characteristics of baboons and macaques are as follows. First, they form large troops containing both males and females. Second, they are omnivorous, feeding on diverse resources including plants, animals, insects, etc. Third, they adapt readily to various environments, from the seashore to high altitudes.

Some Japanese monkeys live high up in the Japanese Alps, reaching $2500 \mathrm{~m}$. They are known to capture and eat birds called rock ptarmigans (Lagopus mutus). They use caves to shelter from heavy snows in winter in the Kurobe valleys (Kashiwagi et al. 2012). The key here is adaptation to various environments. They invaded new habitats and started to eat new and varied items. This reminds me of the case of human evolution and our species' adaptation to new environments. We might be the most extreme case of adapting to various environments on the planet.

Migration is also crucial when we think about adapting to various environments. The baboons in Gorongosa showed me such an example. The troop on the flood plain

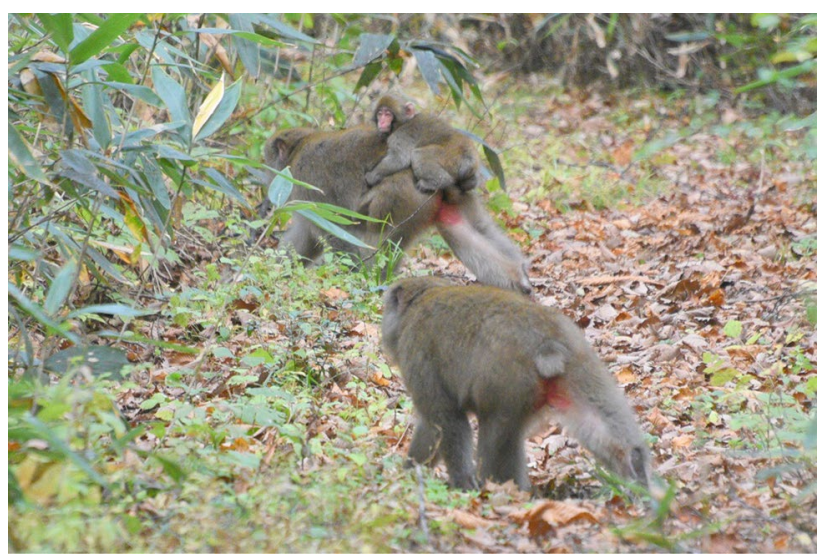

Fig. 3 Japanese monkeys in Sasagamine (photo taken on October 15, 2018 by Shigeru Sugiyama) 

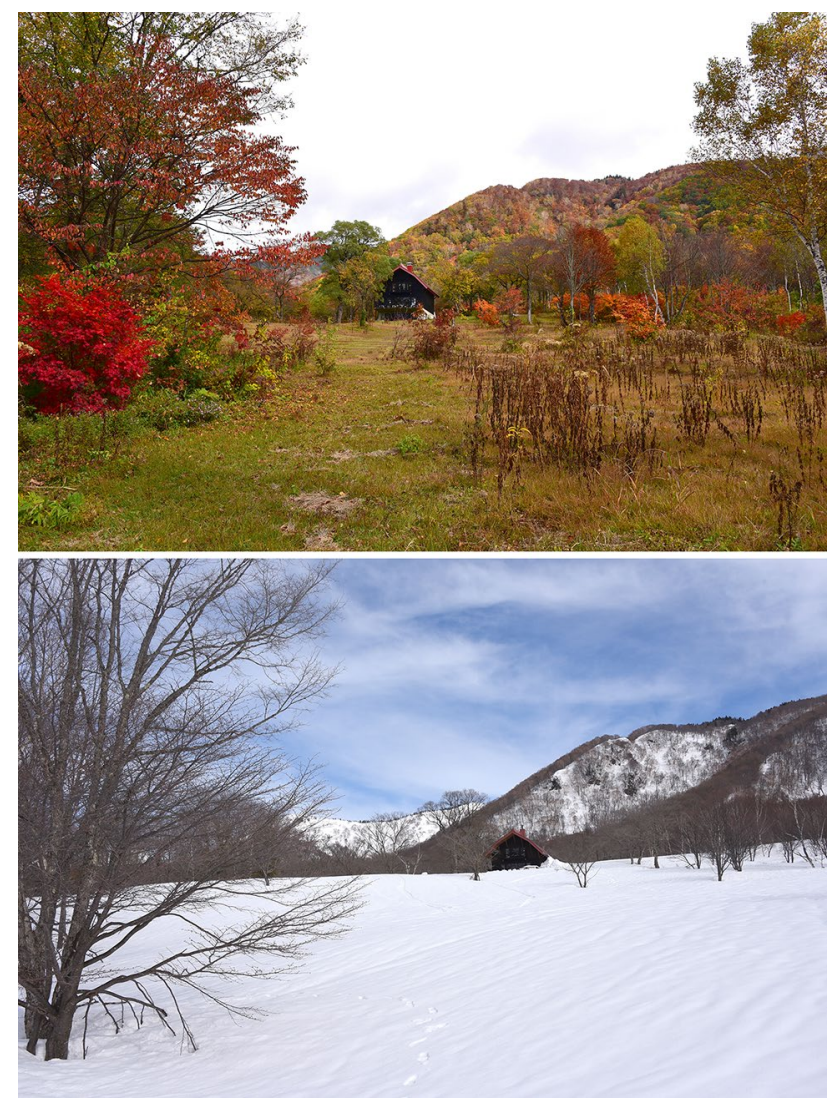

Fig. 4 Sasagamine huette in autumn (top) and winter (bottom). The photos were taken from the same place to get the same image in different seasons: autumn on October 14, 2017 and winter on February 28, 2017 (photos taken by Shigeru Sugiyama)

must change their habitat from the green open land in the dry season to dry, less open habitats in the rainy season. Japanese monkeys in Sasagamine live in their new habitat in the summer, but they have to move down to lower altitudes to avoid the 4-m-deep snow in the winter (Fig. 4). So far, our studies have been focusing on troops of Japanese monkeys that always occupy certain areas. However, it might be worthwhile to focus also on seasonally migrating troops. The latter may give us clues to understanding human evolution from the viewpoint of adaptation to various environments, in association with migration.

Acknowledgements I am grateful to Drs. Susana Carvalho and Dora Biro of Oxford University. They gave me the opportunity to visit Gorongosa. Thanks are due to Prof. Richard Wrangham of Harvard University who was visiting Gorongosa with me at the same time, and Mr. Gregory Carr who welcomed us to the park. Financial support for preparing the manuscript came from MEXT-JSPS Grants \#24000001, \#16H06283; the Japan Society for the Promotion of Science (JSPS) Core-to-core Program CCSN, and the Leading Graduate Program of Primatology and Wildlife Science (U04) to the author. I also thank Dr. Shigeru Sugiyama for guiding me in Sasagamine, and Drs. Jim Anderson and Dora Biro for editing the English text.

\section{References}

Kashiwagi K, Abe Y, Takai M (2012) Cave use by Japanese monkeys living in the deep snow area in Kurobe valley. Primate Res 28:141-153. https://doi.org/10.2354/psj.28.012 (in Japanese)

Matsuzawa T (2017) Horse cognition and behavior from the perspective of primatology. Primates 58:473-477

Matsuzawa T (2018) Hot-spring bathing of wild monkeys in ShigaHeights: origin and propagation of a cultural behavior. Primates 59:209-213

Matsuzawa T, Hasegawa Y, Gotoh S, Wada K (1983) One-trial longlasting food aversion learning in wild Japanese monkeys (Macaca fuscata). Behav Neural Biol 39:155-159

Pelé M, Bonnefoy A, Shimada M, Sueur C (2017) Interspecies sexual behavior between a male Japanese macaque and female sika deer. Primates 58:275-278

Schofield D, McGrew W, Takahashi A, Hirata S (2018) Cumulative culture in nonhumans: overlooked findings from Japanese monkeys? Primates 59:113122

Wada K, Matsuzawa T (1986) A new approach to evaluating troop deployment in wild Japanese monkeys. Int J Primatol 7:1-14

Wilson EO (2014) A window on eternity: a biologist's walk through Gorongosa National Park. Simon \& Schuster Inc, New York

Zhang P, Watanabe K, Tokida E (2007) Habitual hot-spring bathing by a group of Japanese macaques (Macaca fuscata) in their natural habitat. Am J Primatol 69:1425-1430 